\title{
Fraction Crystalline from Electron Powder Patterns of Unlayered Graphene in Solidified Carbon Rain
}

Phil Fraundorf ${ }^{1}$, Melanie Lipp ${ }^{2}$, Tristan Hundley ${ }^{1}$, Chathuri Silva ${ }^{1}$ and Philip Chrostoski ${ }^{1}$

${ }^{1}$ University of Missouri-Saint Louis, St. Louis, Missouri, United States, ${ }^{2}$ University of Stuttgart, Stuttgart, Baden-Wurttemberg, Germany

Elemental carbon at low (e.g. ambient and lower) pressure sublimates to vapor near 4000K, but liquid carbon is reported after laser ablation [1,2]. Some meteoritic carbon particles, whose isotopes fingerprint s-process nucleosynthesis and likely formed in or near red giant star photospheres, show an "unlayeredgraphene" core/graphite-rim structure [3-5] (with HRTEM evidence for faceted pentacones [6]). The cores likely result from super-cooled carbon droplets that nucleated graphene sheets on randomly oriented 5 and 6 member rings. Similar core-rim particles result from slow cooling of carbon vapor in the lab [7], albeit with graphene sheets having smaller coherence widths.

The Debye scattering equation [8] allows one to calculate the interference effect of small randomlyoriented crystalline clusters on otherwise diffuse atomic scattering from a specimen. It is ideal for working with oddly-shaped clusters (like unlayered graphene sheets, and faceted carbon pentacones) because molecule shape effects (like the high-frequency tails on powder diffraction peaks from atom-thick sheets [9]) are automatically taken into account in its predictions.

Electron powder diffraction (and HRTEM) observations of presolar onion cores suggest an average graphene sheet size of about $40 \mathrm{~A}$ coherence width, or $\mathrm{N} \approx 612$ atoms per cluster. Diffraction from the labgrown cores instead suggest sheets with only $12 \mathrm{~A}$ coherence width, or $\mathrm{N} \approx 60$ atoms per cluster. This is not surprising since the small size of our oven interior suggests that condensed particles impact an oven wall within about 10 milliseconds, while containerless cooling of carbon condensate in a red giant photosphere might take place over much longer times (limited only e.g. by radiation pressure ejection).

One way to estimate the fraction crystalline at each peak is to measure three heights at the peak frequency on a model "100\%-crystalline" Debye system with comparable peak width, namely the total peak height $\mathrm{s} 2$, the estimated background height s1 beneath the peak, and the "diffuse-scattering baseline" height $\mathrm{s}_{\mathrm{x}}$. Then on an "experimental profile" measure the observed total peak height $\mathrm{h} 2$ and the corresponding peak background $\mathrm{h}_{1}$ beneath the peak.

One can infer the experimental baseline height $\mathrm{h}_{\mathrm{x}}$ by assuming that the incoherent baseline position is where the model predicts i.e. $\left(\mathrm{h}_{\mathrm{x}}-\mathrm{h}_{1}\right) /\left(\mathrm{h}_{2}-\mathrm{h}_{1}\right)=\left(\mathrm{s}_{\mathrm{x}}-\mathrm{s}_{1}\right) /\left(\mathrm{s}_{2}-\mathrm{s}_{1}\right)$, so that $\mathrm{h}_{\mathrm{x}}=\mathrm{h}_{1}+\left(\mathrm{h}_{2}-\mathrm{h}_{1}\right)\left(\mathrm{s}_{\mathrm{x}}-\mathrm{s}_{1}\right) /\left(\mathrm{s}_{2}-\mathrm{s}_{1}\right)$. The non-crystalline background component $\mathrm{h}_{\mathrm{o}}$ may then be inferred by assuming that the crystallinecomponent peak shape follows the prediction i.e. $\left(\mathrm{h}_{\mathrm{x}}-\mathrm{h}_{\mathrm{o}}\right) /\left(\mathrm{h}_{2}-\mathrm{h}_{\mathrm{o}}\right)=\mathrm{s}_{\mathrm{x}} / \mathrm{s}_{2}$ so that $\mathrm{h}_{\mathrm{o}}=\left(\mathrm{h}_{1} \mathrm{~s}_{2}-\mathrm{h}_{2} \mathrm{~s}_{1}\right) /\left(\mathrm{s}_{2}-\mathrm{s}_{1}\right)$, where any combination of subscripts 1,2 and $\mathrm{x}$ may be used in place of subscripts $\mathrm{x}$ and 2 here. Finally, the fraction crystalline $\mathrm{f}_{\mathrm{x}}$ may be estimated if one divides the inferred crystalline-component baseline by the experimental baseline, i.e. $\mathrm{f}_{\mathrm{x}} \approx\left(\mathrm{h}_{\mathrm{x}}-\mathrm{h}_{\mathrm{o}}\right) / \mathrm{h}_{\mathrm{x}}=\left(\left(\mathrm{h}_{2}-\mathrm{h}_{1}\right) \mathrm{s}_{\mathrm{x}} /\left(\left(\mathrm{h}_{2}-\mathrm{h}_{1}\right) \mathrm{s}_{\mathrm{x}}+\mathrm{h}_{1} \mathrm{~s}_{2}-\mathrm{h}_{2} \mathrm{~s}_{1}\right)\right.$.

This fraction crystalline will be less than one provided that the total peak to (peak-background) height ratio is less in the experimental sample i.e. $\mathrm{h}_{2} / \mathrm{h}_{1}<\mathrm{s}_{2} / \mathrm{s}_{1}$, and hence that the non-crystalline background height $h_{o}$ is positive. By measuring this $f_{x}$ value on a given profile at more than one peak, one might report a mean and a measure of uncertainty as well. For the experimental (Fig. 1) and model (e.g. Fig. 2) pattern 
profiles, the fraction of atoms in unlayered graphene sheets about 600 atoms in size (presolar) and 60 atoms in size (homegrown) is estimated to be about $40 \%$ and $12 \%$, respectively.

The "time at temperature" (e.g. in the $3000 \mathrm{~K}$ range) needed to rearrange liquid atoms, so that nearly half of them are in graphene sheets about 600 atoms in size, would tell us both about the way that carbon atoms on earth were condensed and ejected from red giant atmospheres, and about the challenges we might face in trying to create similar materials on earth e.g. for their potentially-interesting diffusion barrier properties. The ratio between crystalline fraction $f_{x}$ and the number of atoms per sheet also be used to tell us the number of sheets per atom (or per cc, or per gram, for a given density), and (depending on whether they are nucleated on pentagonal or hexagonal loops) the number of sheet nuclei which have managed to seed growth to the average final size during the cooldown process. These numbers in turn are being compared with ab-initio and semi-empirical predictions of loop formation in a solidifying carbon melt [10], with the goal of refining cluster-kinetic and analytical models of nucleation and growth in the days ahead.

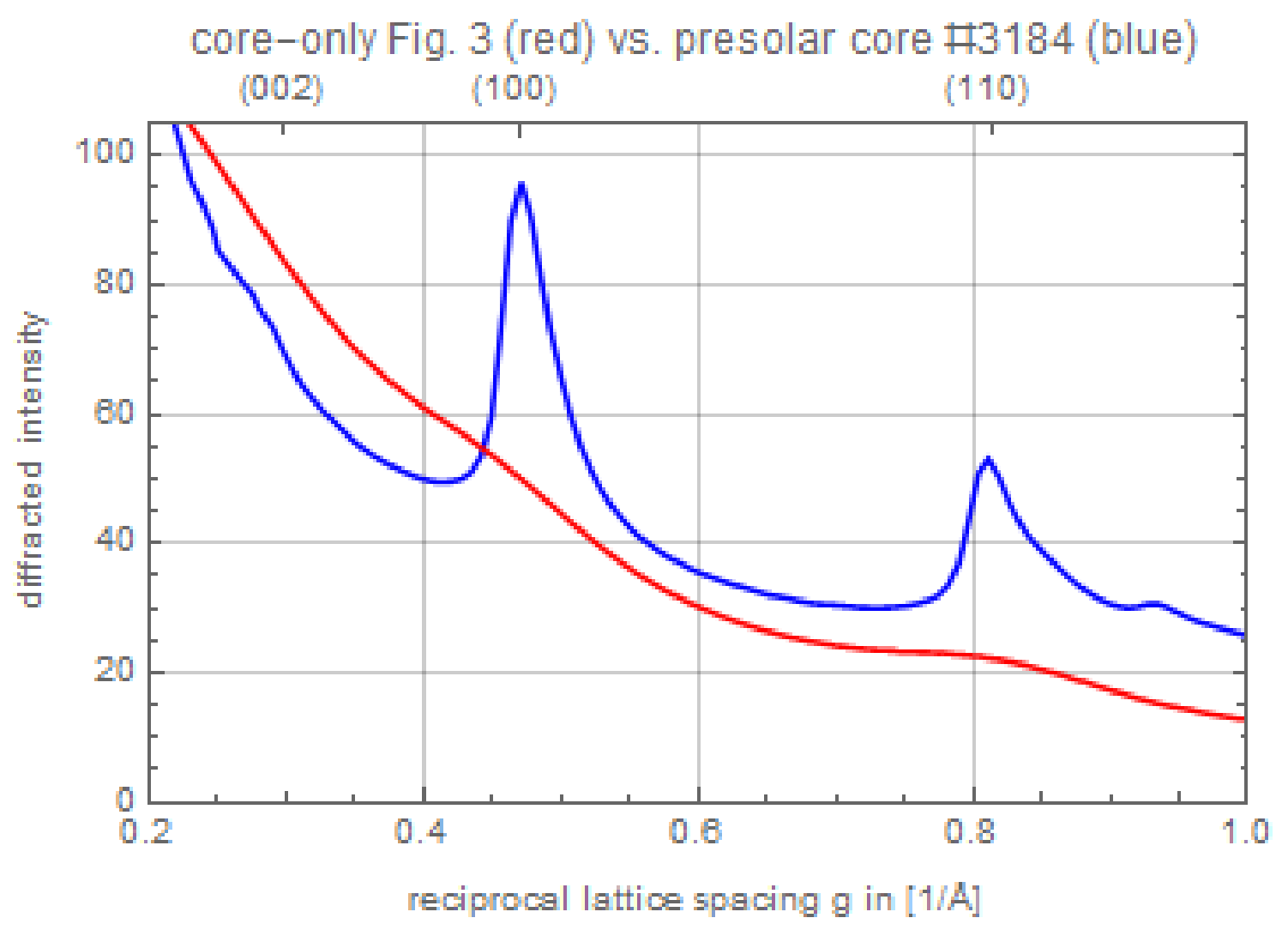

Figure 1. Presolar (blue) vs. homegrown (red) diffraction profiles, showing the almost complete absence of graphite (002). 


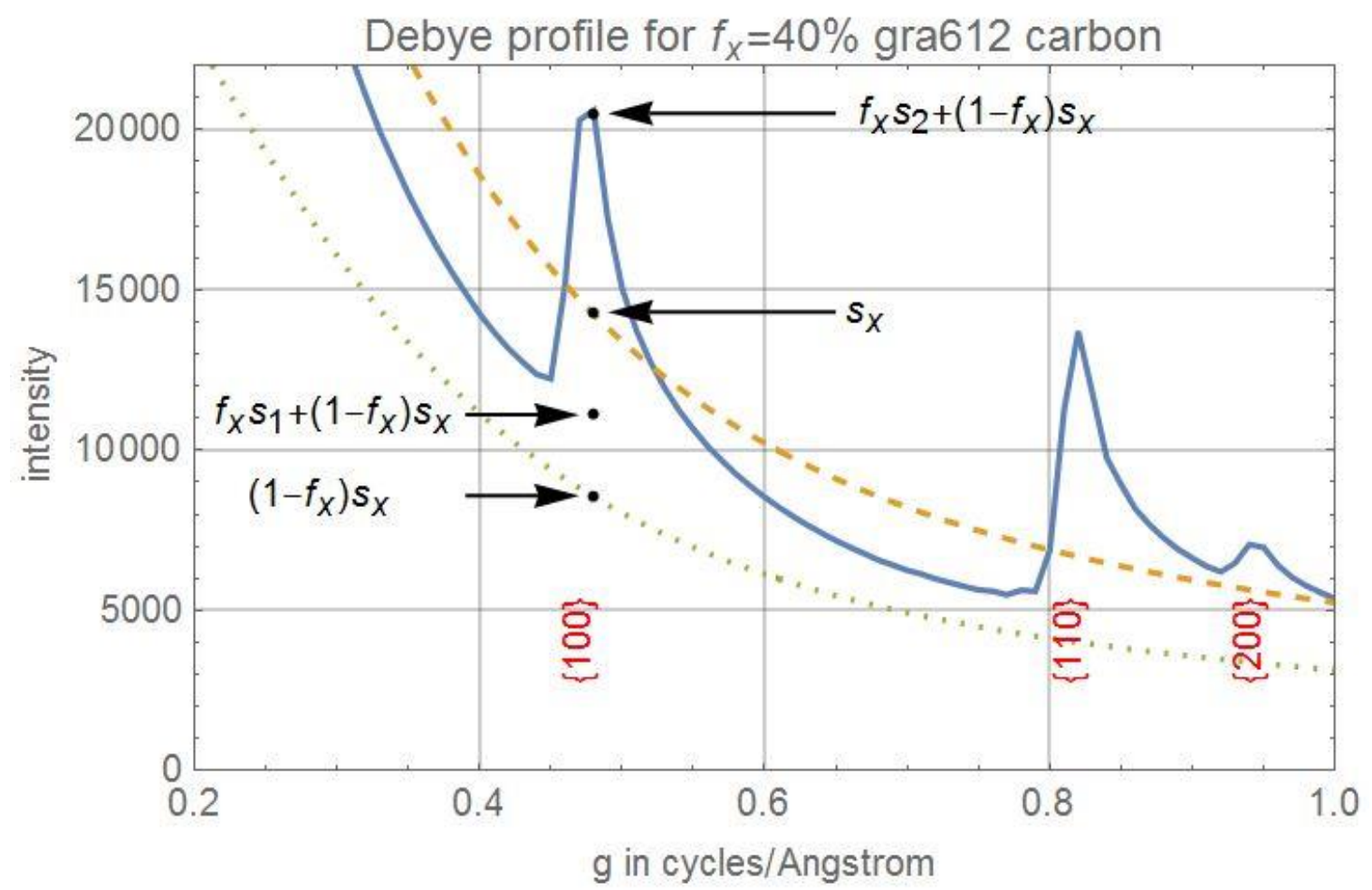

Figure 2. Debye profile of carbon, $40 \%$ of whose atoms are in the form of randomly-oriented and unlayered graphene sheets about 600 atoms in size, with the remaining atoms (at interstices) that scatter incoherently. The diffuse scattering baseline is dashed.

References

[1] D. Kasuya, M. Yudasaka, K. Takahashi, F. Kokai, and S. Iijima "Selective Production of Single-Wall Carbon Nanohorn Aggregates and Their Formation Mechanism" (2002) J. Phys. Chem B 106, 4947.

[2] W.A. De Heer, P. Poncharal, C. Berger, J. Gezo, Z.M. Song and J. Bettini et al., "Liquid carbon, carbon-glass beads, and the crystallization of carbon nanotubes" (2005) Science 307:5711, pp. 907-910.

[3] T. Bernatowicz, R. Cowsik, P. C. Gibbons, K. Lodders, B. F. Jr., S. Amari, and R. S. Lewis (1996) "Constraints on stellar grain formation from presolar graphite in the Murchison meteorite", Astrophysical Journal 472, 760.

[4] P. Fraundorf and Martin Wackenhut, "The core structure of pre-solar graphite onions" (2002) Ap. J. Lett. 578(2) L153-156

[5] T. Kevin Croat, Thomas J. Bernatowicz, and Tyrone L. Daulton (2014) "Presolar graphitic carbon spherules: Rocks from stars" Elements 10, 441-446.

[6] Eric Mandell, "Electron Beam Characterization of Carbon Nanostructures" (2007) Ph.D. Dissertation at UM-St Louis/Rolla.

[7] P. Fraundorf, Tristan Hundley and Melanie Lipp (2019) "Synthesis of unlayered graphene from carbon droplets: In stars and in the lab", HAL-02238804, google site.

[8] B. E. Warren (1969/1990) X-ray diffraction (Addison-Wesley, Reading MA/Dover, Mineola NY).

[9] B. E. Warren (1941) "X-ray diffraction in random layer lattices", Phys. Rev. 59(9), 693.

[10] Chathuri Silva, P. Fraundorf, E. H. Majzoub, and P. Chrostoski (2020) "DFT studies of graphene in carbon droplets condensed in stellar atmospheres", MRS Spring Meeting (Phoenix AZ). 